coefficient of the molecular orbital resonance integral in the expression for the energy of the lowest unoccupied orbital. A plot of $E$ against $a$ is shown in the accompanying figure. The approximately linear relationship between $E$ and $n$ verifies the postulated mechanism. Further evidence may be obtained by considering the overall equilibrium between $R$ and $R \mathrm{H}_{2}$. If this were the potential-determining process, there should exist a relationship between the potential and the difference in resonance energies of $R \mathrm{H}_{2}$ and $R$. No such relationship exists.

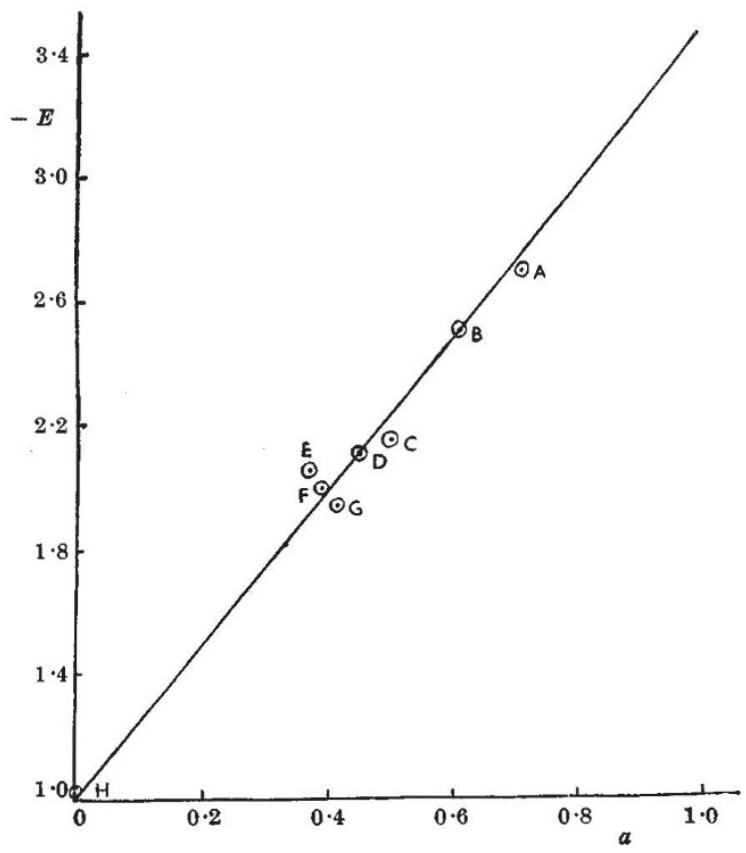

The reduction potentials of some hydrocarbons as a function of $a$. The substances referred to are: $\boldsymbol{A}$, diphenyl; $\boldsymbol{B}$, naphthalene ; $C$, stilbene; $D$, pyrene ; $E$, tetraphenyl ethylene ; $F, 1: 4$ diphenyl butadiene; $G$, anthracene; and $H$, triphenyl methyl.

On the basis of the above theory, the difficulty of reduction of an isolated double bond and of benzene may be understood, for in these cases a very large negative potential would be required. In the former case polarographic reduction differs from catalytic reduction ${ }^{3}$.

Reduction by dissolving metals may also be discussed on these lines. Two possible mechanisms exist in this case,

$$
\begin{aligned}
& R+\mathrm{H}^{+} \rightarrow R \mathrm{H}^{+} \\
& R \mathrm{H}^{+}+2 e^{i} \rightarrow R \mathrm{H}^{-} \\
& R \mathrm{H}^{-}+\mathrm{H}^{+} \rightarrow R \mathrm{H}_{2}
\end{aligned}
$$

as suggested by Burton and Ingold ${ }^{4}$; or

$$
\begin{aligned}
& R+2 \mathrm{Na} \rightarrow R^{-}+2 \mathrm{Na}+ \\
& R^{=}+2 B \mathrm{H} \rightarrow R \mathrm{H}_{2}+2 B^{-}
\end{aligned}
$$

as proposed by Zieglers. In addition, there is the possibility in the former scheme of the consecutive addition of the electrons. Two problems arise in these cases, namely, the ease of reduction, and the products formed. The former may be discussed along the lines suggested above. For a discussion of the latter, the electron structure of $R \mathrm{H}^{-}$and $R^{\mathbf{m}}$ will have to be examined. The position of addition of the protons will be determined by the charge distribution of the ions.

It is hoped shortly to publish a fuller account of this work.

Sir William Ramsay and

Allan Maccoll

Ralph Forster Laboratories, University College,

London, W.C.I. Aug. 10.

${ }^{1}$ Wazownek et al., J. Amer. Chem. Soc., 64, 1765, 2365 (1942); 68 2541 (1946).

Hückel, Int. Conf. on Physics, 9 (London, 1934).

Maccoll, Nature [168, 138 (1949)].

- Burton and Ingold, J. Chem. Soc., 2022 (1929).

'Ziegler, Z. angew. Chem., 49, 449 (1936). See also Birch, Faraday Society Discussion on the Labile Molecule, 246 (1947).

\section{Electron Distribution in Substituted Styrenes Derived from Infra-Red Spectra}

Is a recent communication ${ }^{1}$, it was pointed out that the abnormalities in the physical properties and polymerization characteristics of vinyl mesitylene could be ascribed to steric hindrance by the methyl groups in the ortho positions; it was mentioned that a study of the infra-red spectra of the styrenes revealed differences that supported the hypothesis that this 'blocking' affected the electronic structure.

In the light of recent developments in the normal co-ordinate treatment of group vibrations ${ }^{2}$, a more precise interpretation of the infra-red results may now be given. The key $\delta_{\mathrm{CH}_{3}}-$ band $^{2}$ in styrene lies at $913 \mathrm{~cm} .^{-1}$. The shifts from this frequency in $m$. and $p$-methyl styrenes and in 5-vinyl-pseudocumene have values of $-5,-4$ and $-5 \mathrm{~cm}^{-1}$ respectively; the shift in vinyl-mesitylene is $+12 \mathrm{~cm} .^{-1}$. The absolute magnitudes of $\delta_{\mathrm{CH}}$, indicate that there is a resultant negative charge on the terminal carbon atom of the vinyl group in all five compounds, which will be associated with $m$-substitution in the phenyl nucleus. The methyl groups in the three compounds with negative increments give rise to a small additional negative charge over and above that due to conjugation with phenyl; presumably the normal effect found in propylene ${ }^{2}$ is transmitted via the conjugated system. In vinyl mesitylene, the relative instability of planar structures inhibits the resonance effect, and, in spite of the presence of three methyl groups, the increment is positive, though the electron distribution is still such as to give rise to $m$-direction.

I wish to express my gratitude to Trinidad Leaseholds, Ltd., for allowing me access to the infra-red data used in this note.

39a Palliser Road,
London, W.14.

P. TORKINGTON

Aug. 22.

${ }^{2}$ Buck, F. R., Kennedy, G. T., Morton, F., and Tanner, E. M., Nature, $162,103(1948)$

- Torkington, P., Proc. Roy. Soc. (in the press); Nature [162 370 (1948) ].

\section{Thermochemistry of Acetylene and Allylene and the Valency of Carbon}

Recentux, Long and Norrish ${ }^{1}$ have shown that when the energy associated with the tetravalent state of carbon is systematically taken into account it is possible to bring order into the thermochemistry of carbon. They support the value of $190 \mathrm{kcal}$. for the latent heat of sublimation of graphite into free atoms 